\title{
Inhibición de butirilcolinesterasa en dos perros intoxicados y confirmación analítica de carbofuran como agente causal
}

\author{
Ferré, D.M.,2; Saldeña, E.L. ; Albarracín, L. ${ }^{1}$; Neuilly, V. ${ }^{1,3}$; Gorla, N.B. ${ }^{1,2}$ \\ ${ }^{1}$ Laboratorio de Genética, Ambiente y Reproducción, Facultad de Ciencias Veterinarias y Ambientales, \\ Universidad Juan Agustín Maza, acceso este, lateral sur 2245, Mendoza, Argentina. ${ }^{2}$ CONICET. ${ }^{3}$ Cuerpo \\ Médico Forense y Criminalístico, Mendoza. Tel. 261-4056222, E-mail: noragorla@gmail.com
}

\begin{abstract}
Resumen
Ferré, D.M.; Saldeña, E.L.; Albarracín, L.; Neuilly, V.; Gorla, N.B.: Inhibición de butirilcolinesterasa en dos perros intoxicados y confirmación analítica de carbofuran como agente causal. Rev. vet. 26: 1, 43-48, 2015. La mortandad de animales domésticos y silvestres debido a intoxicaciones por plaguicidas es internacionalmente elevada, en la mayoría de los casos por exposición a insecticidas. Los más comúnmente citados son los anticolinesterásicos organofosforados y carbamatos. Los perros y gatos mascotas son buenos recursos para alertar sobre el uso de venenos en el medio natural y los posibles riesgos para el hombre con el que comparten el ambiente. En dos perros con signos de intoxicación, el objetivo del presente trabajo fue efectuar estudios complementarios de laboratorio para identificar con certeza la causa de las intoxicaciones. Se reporta el manejo clínico, la aproximación diagnóstica a través de la determinación en plasma de butirilcolinesterasa $(\mathrm{BChE})$ por espectrofotometría y la confirmación del tóxico por cromatografía líquida de alta precisión masa- masa. La actividad BChE de cada perro fue de $227 \mathrm{UI} / \mathrm{L}$, lo que significa una inhibición de más del $90 \%$ de la actividad basal. La presencia de carbofuran en plasma y contenido gástrico corroboró la causa de las intoxicaciones. Los plaguicidas agrícolas inhibidores de colinesterasa, especialmente los carbamatos, son la principal causa de envenenamiento en animales. Por tal motivo se sugiere como herramienta de diagnóstico la medición de $\mathrm{BChE}$ en animales intoxicados, así como bregar por mayores controles en la comercialización de plaguicidas ricos en ingredientes activos con bajas DL50, como el caso del carbofuran. En salud pública debería exigirse la denuncia obligatoria y registro centralizado de las intoxicaciones intencionales o accidentales por plaguicidas agrícolas en animales, cuyo mal uso puede atentar incluso contra la vida de seres humanos.
\end{abstract}

Palabras clave: perro, intoxicación, carbofuran, colinesterasa, HPLC.

\begin{abstract}
Ferré, D.M.; Saldeña, E.L.; Albarracín, L.; Neuilly, V.; Gorla, N.B.: Butyrilcholinesterase inhibition and carbofuran analytical confirmation in two intoxicated dogs. Rev. vet. 26: 1, 43-48, 2015. Domestic and wild animals' death due to pesticide poisoning is of worldwide concern and in most of the cases is a consequence of the exposure to insecticides. Organophosphorus and carbamates anticholinesterase compounds are frequently implicated. Pet dogs and cats are considered good resources to warn about the presence of poisons in the environment and the potential risks they represent to human beings. The aim of this work was to perform complementary laboratory studies in two dogs with signs of intoxication in order to identify the possible cause. Diagnostic approach through plasmatic butyril cholinesterase (BChE) and HPLC determination are presented. BChE activity of each canine was $227 \mathrm{IU} / \mathrm{L}$ thus meaning an inhibition of more than $90 \%$ of the basal activity of the enzyme. The presence of carbofuran in plasma and gastric contents as the cause of poisoning was confirmed. Cholinesterase inhibitors pesticides, especially carbamates, are the leading cause of poisoning in animals. The determination of $\mathrm{BChE}$ activity in intoxicated animals as a diagnostic tool is emphasized. Also, strong regulations on the commercialization of pesticides with low LD50 such as carbofuran as well as the report of both intentional and accidental intoxications are mandatory in order to avoid further cases in animals and possible cases in humans.
\end{abstract}

Key words: dog, intoxication, carbofuran, cholinesterase, HPLC. 


\section{INTRODUCCIÓN}

Los perros envenenados intencional o accidentalmente son una realidad en los consultorios veterinarios, con profusión de información en cuanto a zonas geográficas, causas hipotéticas y alertas de los vecinos, con un número de animales alarmante, que llega en algunos casos a dos centenares. Esta realidad está acompañada por la ausencia de denuncia obligatoria profesional y de registros gubernamentales habilitados a tal fin. Internacionalmente, la casuística de mortandad de animales domésticos y silvestres debido a plaguicidas es elevada. Los animales domésticos como perros y gatos se consideran buenos recursos para alertar sobre el uso de venenos en el medio natural ${ }^{17}$.

En una publicación reciente se efectúa una revisión de los registros de intoxicaciones de animales de compañía en países europeos. Los perros son la especie más comúnmente envenenada, particularmente los animales jóvenes. La mayoría de los casos de intoxicación en animales de compañía resulta de la exposición a insecticidas, si bien los rodenticidas asumen un riesgo significativo. Las intoxicaciones con herbicidas, metales, productos del hogar y drogas de uso veterinario y humano son de presentación esporádica ${ }^{1}$.

Los insecticidas comúnmente involucrados en intoxicaciones animales son los anticolinesterásicos organofosforados (OP) y carbamatos (CB) ${ }^{8,9,17}$. Los signos de intoxicación aguda por OP son la consecuencia de la sobre-estimulación colinérgica por inhibición de la colinesterasa (ChE) con efecto en los músculos, glándulas y/o células nerviosas, que puede ser agrupada en 3 categorías: signos muscarínicos, nicotínicos y centrales, lo que incluye al sistema nervioso periférico y central. Los signos muscarínicos son usualmente los primeros en aparecer e incluyen hipersalivación, miosis, micción espontánea, diarrea, vómitos, cólicos y disnea debido a secreciones bronquiales aumentadas y broncoconstricción. Los efectos nicotínicos incluyen fasciculaciones musculares, rigidez muscular y debilidad. Los efectos centrales incluyen nerviosismo, ataxia y convulsiones ${ }^{9,21}$.

Luego de la sintomatología aguda, se puede presentar tanto en el hombre como en animales el síndrome intermedio y/o neuropatía retardada entre las $24-48 \mathrm{~h} \mathrm{y}$ las 2-3 semanas siguientes a la intoxicación aguda respectivamente. El síndrome intermedio cursa con debilidad muscular de los miembros anteriores y músculos de la respiración, en tanto que la neuropatía retardada lo hace con debilidad muscular simétrica, ataxia y déficit propioceptivo consciente, principalmente de los miembros pélvicos ${ }^{2,11}$.

Los signos de intoxicación por CB se describen con el acrónimo "slud": salivación, lagrimeo, "urination" y diarrea. Debido a que los CB no atraviesan la barrera hematoencefálica con facilidad, los signos de sistema nervioso central son limitados. La muerte resulta de la falla respiratoria debido a la broncoconstricción que conduce a la secreción traqueobronquial y edema pulmonar ${ }^{19}$. Las intoxicaciones por CB no presentan síndrome intermedio ni neuropatía retardada, debido a que la inhibición de colinesterasa es reversible.

En las intoxicaciones con inhibidores de colinesterasa, un aporte diagnóstico importante es la determinación de la actividad ChE en sangre y cerebro. La sintomatología es dependiente de la disminución de la acetilcolinesterasa (AChE) neuronal, aunque también se deprime la actividad de la AChE eritrocitaria y la pseudocolinesterasa, colinesterasa plasmática o butirilcolinesterasa (BChE). La AChE hidroliza al neurotransmisor acetilcolina $(\mathrm{ACh})$ produciendo colina y ácido acético, mientras que la $\mathrm{BChE}$ hidroliza a la $\mathrm{ACh}$ más lentamente y a otros ésteres ${ }^{5}$. Los signos clínicos son visibles cuando la actividad de la ChE cerebral está inhibida $>70 \%$ y la BChE $>50 \%{ }^{20}$.

Las sintomatologías agudas de intoxicaciones por OP y CB son tratadas con atropina y también benzodiazepinas para controlar el estado de excitación causado por la intoxicación. Para intoxicaciones con OP se utilizan oximas (obidoxima, pralidoxima) para reactivar la actividad $\mathrm{ChE}^{13,20}$.

En esta comunicación se reporta el manejo clínico de dos perros intoxicados, la aproximación diagnóstica a través de la inhibición de ChE y la confirmación analítica de la presencia de carbofuran.

\section{MATERIAL Y MÉTODOS}

Animales. Caso 1: perro macho de raza labrador de aproximadamente 1 año de edad y $30 \mathrm{~kg}$, residente de zona noreste de la Provincia de Mendoza. Fue llevado a la consulta con hiperventilación, mioclonías, sialorrea, diarrea y congestión de mucosas ocular y bucal. Los propietarios relataron haber fumigado los cultivos de hortalizas y olivos de la finca donde habitaba el perro y que el animal se encontraba libre al momento de la aplicación de los plaguicidas.

Caso 2: perro macho de raza dálmata de aproximadamente 5 años de edad y $23 \mathrm{~kg}$, residente de zona urbana del norte de la Provincia de Mendoza. Fue llevado a la consulta con extrema debilidad, hipotermia, desorientación, dolor abdominal y nistagmos. Posteriormente comenzó con vómitos sanguinolentos frecuentes. Los propietarios informaron que salía a pasear todos los días sin compañía y que fue encontrado en una plaza, visiblemente descompensado y llevado a la veterinaria. La necropsia fue realizada tras la muerte del animal.

Tratamientos. El caso 1 fue tratado con sulfato de atropina $0,5 \mathrm{mg} / \mathrm{kg}$ vía subcutánea (SC) y luego fluidoterapia de mantenimiento vía endovenosa (EV) con solución de cloruro de sodio al $0,9 \%$ hasta la estabilización del paciente. Luego se continuó con administración de sulfato de atropina EV, hasta la disminución de los signos. Para controlar las mioclonías y lograr la relajación del animal, se administraron $0,5 \mathrm{mg} / \mathrm{kg}$ de diazepan EV a una concentración de $10 \mathrm{mg} / \mathrm{ml}$. La 
atropina fue administrada por $24 \mathrm{~h} 0,5 \mathrm{ml} \mathrm{SC}$ cada $12 \mathrm{~h}$, carbón activado 2 comprimidos cada $12 \mathrm{~h}$ y $3 \mathrm{ml}$ cada $12 \mathrm{~h}$ de protector hepático a base de sorbitol, metionina y complejo vitamínico B por $72 \mathrm{~h}$ vía oral. La extracción de sangre periférica fue realizada con heparina y enviada refrigerada al laboratorio de toxicología. El paciente fue controlado a las $24 \mathrm{~h}$ de realizada la consulta. El caso 2 recibió fluidoterapia EV con solución de cloruro de sodio al $0,9 \%$ y fue administrada atropina $0,5 \mathrm{mg} / \mathrm{kg}$. El $50 \%$ de la dosis total se administró vía EV y la mitad restante vía SC. La extracción de sangre fue realizada con heparina. La sangre y el contenido gástrico obtenido en la necropsia fueron enviados al laboratorio de toxicología.

Medición de la actividad butirilcolinesterasa. Para la determinación de la $\mathrm{BChE}$ se centrifugaron las muestras de sangre 10 minutos a $1.500 \mathrm{rpm}$ para obtener plasma, y fue utilizado el kit GT lab, Rosario, Argentina, método cinético $405 \mathrm{~nm}, \mathrm{pH}$ 7,7. Este kit determina la concentración de la enzima $\mathrm{BChE}$, mediante butirilcolina como sustrato. La determinación enzimática fue realizada dentro de las $24 \mathrm{~h}$ de la extracción sanguínea.

Detección analítica de plaguicidas. Sangre y contenido gástrico fueron tratados con acetonitrilo frío $(1: 1,5)$ y centrifugados durante $5 \mathrm{~min}$ a $3.000 \mathrm{rpm}$. El sobrenadante obtenido fue centrifugado durante $2 \mathrm{~min}$ a $12.000 \mathrm{rpm}$, luego mantenido a $-20^{\circ} \mathrm{C}$ durante $20 \mathrm{~min}$. El método de detección utilizado fue cromatografía líquida de alta precisión (HPLC), equipo Agilent 1200 con bomba binaria y muestreado automático equipado con detector masa/masa Api2000 de Applied Biosystems. Condiciones de trabajo: fase móvil formiato de amonio $1 \mathrm{M}, \mathrm{pH}$ 6,3-6,8; $1 \mathrm{ml} / \mathrm{min}$, columna Kromasyl 100-3-5 C18; 50x2,1; E 63958, modo LC Sync, id 17,505, fuente de iones: turbo spray, modalidad de escaneo: MRM, polarización: positiva. Todos los solventes utilizados fueron calidad HPLC Merck. La solución testigo de plaguicida fue furadan $10: 1 \mu \mathrm{g} / \mathrm{ml}$ de principio activo, volumen de inyección: $20 \mu 1$.

\section{RESULTADOS}

En ambos perros la actividad BChE fue de 227 UI/L. La pericia toxicológica en ambos animales evidenció la presencia de carbofuran en sangre del caso 1 y en contenido gástrico del caso 2. En el cromatograma de la Figura 1, a modo ilustrativo, se muestran los resultados del caso 2 .

Posteriormente al tratamiento el paciente caso 1 se estabilizó, normalizó la respiración y disminuyó la sialorrea y mioclonías. Una vez lograda la remisión de los signos fue indicado el alta con medicación ambula-
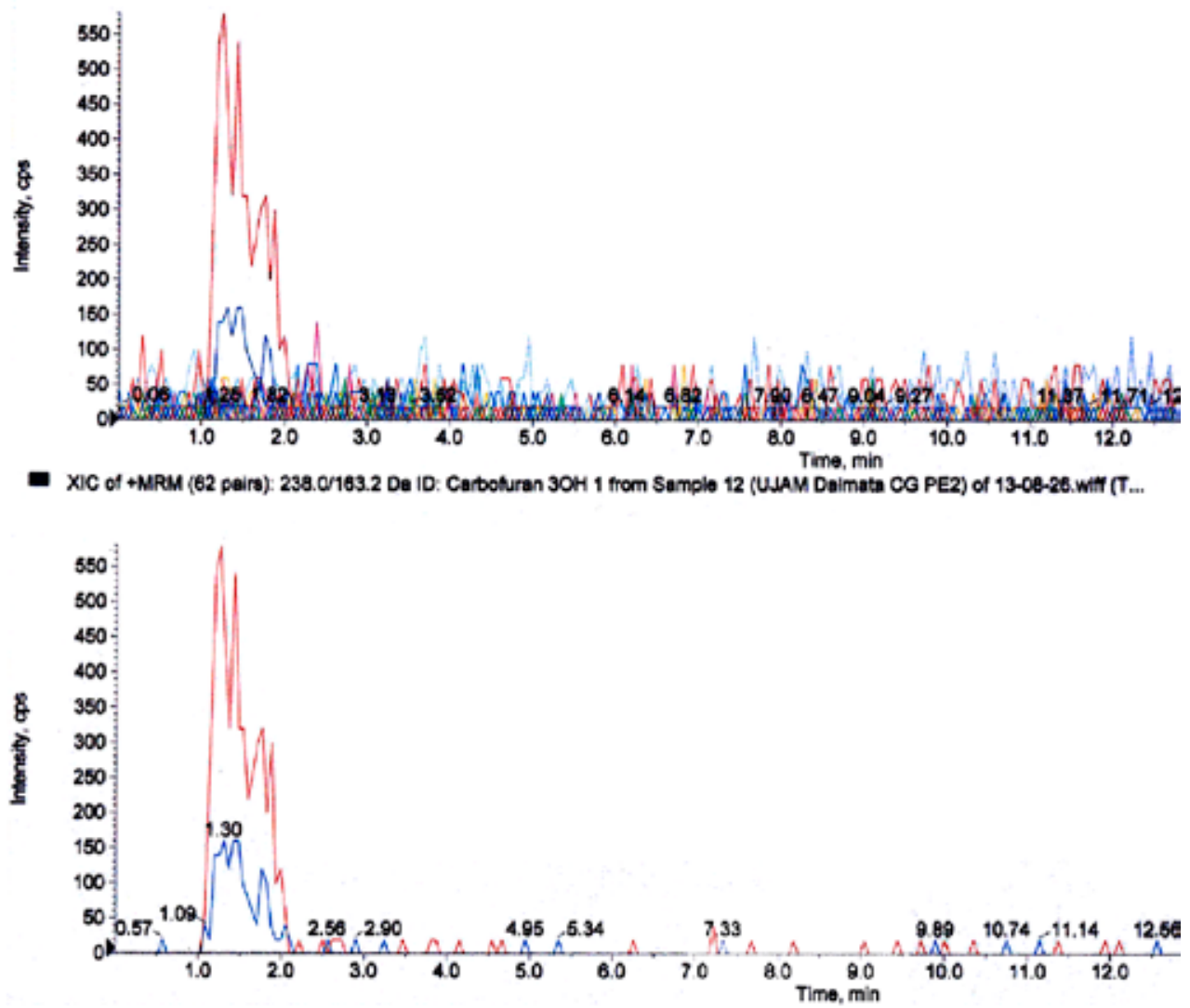

Figura 1. Cromatogramas (HPLC, detector masa-masa) de contenido gástrico de un perro con signos compatibles de intoxicación por anticolinesterásicos (caso 2). Arriba: separación cromatográfica. Abajo: detección por espectrometría masa-masa utilizando ionización por turbospray en polaridad positiva. Los dos picos alrededor de 1,30 min corresponden a los pares iónicos de carbofuran. 
toria.El animal logró superar la intoxicación con asistencia médica. El paciente caso 2 no logró estabilizarse, continuó con vómitos y murió a las 6 h de llegar a la consulta. La necropsia reveló lesiones macroscópicas hemorrágicas en páncreas e hígado y contenido estomacal sanguinolento.

\section{DISCUSIÓN}

El carbofuran es un CB de acción sistémica y de contacto, de uso insecticida y nematicida en agricultura. Está incluido en la lista de plaguicidas altamente peligrosos ${ }^{16} \mathrm{y}$ en la clasificación de toxicología aguda de la Organización Mundial de la Salud es categoría Ib, producto altamente tóxico ${ }^{23}$. El uso de determinados plaguicidas en intoxicaciones intencionales en animales, se debe al tipo de agricultura de la región, conocimiento popular de la toxicidad del principio y a su disponibilidad en el mercado local ${ }^{15}$.

En Argentina el carbofuran está prohibido en peras y manzanas por decreto $2121 / 90$, y cancelada la inscripción y la autorización de uso y comercialización por disposición 2367/2006 ${ }^{18}$. A pesar de esta disposición, en los resultados de 73 entrevistas realizadas en el área rural sureste de Mendoza, aproximadamente el $10 \%$ de los entrevistados relató que aún usaba carbofuran. Los perros intoxicados provenían de las zonas norte y noreste de la provincia. A nivel mundial, es uno de los insecticidas mas utilizados para envenenar animales $6,7,17$ y se han registrado intoxicaciones y muertes (accidentes laborales, suicidios, asesinatos) en varios países ${ }^{12}$.

El carbofuran se comercializa en diferentes formulaciones que rondan el $10-33 \%$ del principio activo y la DL50 en perros es de $19 \mathrm{mg} / \mathrm{kg}^{8}$ por lo que en el caso del perro muerto por intoxicación (23 $\mathrm{kg}$ de peso), el volumen que se necesita para lograr ese efecto es solamente alrededor de $3 \mathrm{ml}$. Los datos de intoxicación por carbofuran en ratas juveniles (días 11 y 17 postnatal) demostraron que pequeñas diferencias en la dosis $(0,1 \mathrm{a} 0,3 \mathrm{mg} / \mathrm{kg})$ pueden cambiar significativamente la inhibición de $\mathrm{AChE}$ cerebral y eritrocitaria desde la ausencia de signos clínicos $(0,1 \mathrm{mg} / \mathrm{kg})$ hasta temblores y actividad motora disminuida $(0,3 \mathrm{mg} / \mathrm{kg})$. Pequeñas diferencias en los niveles de exposición pueden tener consecuencias adversas riesgosas, incluso para seres humanos. Para el caso del carbofuran la diferencia entre la cantidad de alimento contaminado que puede ser consumido en forma segura sin efectos adversos y la cantidad que provee una dosis tóxica excede los niveles mínimos de seguridad ${ }^{10}$.

La inhibición de ChE es una característica distintiva de los plaguicidas OP y CB. Los descensos de las actividades BChE y/o AChE confirman la absorción de estos plaguicidas. Es intersante recordar que sus mecanismos de toxicidad son los mismos para insectos y mamíferos ${ }^{21}$. Los insecticidas anticolinesterásicos causan un complejo con la colinesterasa al unirse covalentemente con el grupo hidroxilo de la serina en el sitio activo, lo que causa la inhibición de ChE y la acu- mulaciónde ACh en las sinapsis colinérgicas del sistema nervioso central, muscarínico y nicotínico ${ }^{13}$.

La reversibilidad del complejo, que puede hidrolizarse espontáneamente, depende del grupo sustituyente del plaguicida. Con los OP puede ocurrir un envejecimiento del complejo enzima-inhibidor en minutos u horas, que es el refuerzo del complejo por la pérdida de un grupo alquilo del aducto forforilado, evitando la reactivación enzimática ${ }^{20}$. Cuando la $\mathrm{AChE}$ es inhibida en forma irreversible, la restauración de su actividad depende de la síntesis de nuevas moléculas de enzima ${ }^{3}$. Los CB tienen la propiedad de que el complejo enzimainhibidor permite la reactivación de la enzima en no más de $24 \mathrm{~h}$, en poco tiempo dejan la enzima libre, por lo que se consideran inhibidores reversibles ${ }^{3,20}$. Los CB más tóxicos son aquellos que mejor se acoplan al centro activo de la enzima (ejemplos: carbofuran, aldicarb, metomil).

La depresión de ChE comienza por lo general inmediatamente después de producirse una absorción significativa de los inhibidores, o dentro de las 24 horas siguientes. La BChE se deprime y recupera antes que la AChE. El descenso de la primera persiste por varios días o pocas semanas; en cambio, la AChE permanece deprimida hasta 1-3 meses. Esta propiedad puede ser usada como indicador del grado de exposición si la actividad de la enzima en sangre es determinada secuencialmente. El muestreo realizado al menos en dos ocasiones puede ser usado para establecer las variaciones intra e interindividuales. La determinación de la actividad tiene óptima significancia cuando es comparada con los valores normales de ese individuo. En la práctica son usados orientativamente niveles de referenciade BChE: $5071 \pm 891 \mathrm{UI} / \mathrm{L}^{4}$. Los perros intoxicados de este trabajo tuvieron una sola medición de BChE, pero mostraron una inhibición mayor al $90 \%$ de su actividad, con lo que se asume el diagnóstico confirmatorio de intoxicación por OP o CB.

Para efectuar el diganóstico diferencial de intoxicacion por $\mathrm{OP}$ o $\mathrm{CB}$, sería necesario realizar por lo menos una segunda determinación de la actividad $\mathrm{BChE}$ en el animal $24 \mathrm{~h}$ con poterioridad a la primera, y poder evaluar si se mantiene el mismo porcentaje de actividad (OP) o si ha aumentado (CB) ${ }^{14}$. En las intoxicaciones por OP tanto en el hombre como en los perros, puede presentarse sintomatología correspondiente a crisis aguda colinérgica, neuropatía intermedia o síndrome intermedio y neuropatía retardada. El tratamiento con atropina no revierte la neuropatía intermedia, la cual provoca dificultad respiratoria y debe ser tratada con ventilación asistida. El uso de pralidoxima, que revierte la union iónica inicial del OP es reservado para la presentación aguda de la intoxicación ${ }^{11}$.

Es necesario también realizar el diagnostico diferencial con patologías o situaciones que pueden presentarse con similar sintomatología, tales como síndrome convulsivo, estado de coma, intoxicaciones con fluoracetato de sodio, hidrocarburos clorados, depresores del sistema nervioso central, enfermedad diarreica aguda, 
edema pulmonar agudo, insuficiencia cardíaca congestiva e hiperreactividad bronquial ${ }^{14}$.

Si el animal muere, la necropsia es esencial a fin de obtener tejidos para el análisis químico y también para estudios bacterianos y virales. Muchos diagnósticos toxicológicos dependen no solo de los residuos del tóxico sino también de las lesiones compatibles con otras enfermedades capaces de causar efectos clínicos similares. Aún cuando un animal individual no se vea beneficiado por la confirmación del laboratorio toxicológico, los resultados pueden proteger a otros animales o seres humanos de sustancias tóxicas o diagnósticos incompletos ${ }^{21}$. En nuestro estudio, la necropsia del perro fallecido (caso 2) reveló lesiones macroscópicas hemorrágicas en páncreas e hígado y contenido estomacal sanguinolento compatibles con intoxicación.

La detección analítica de plaguicidas es un recurso más costoso que la medición de $\mathrm{BChE}$. El método aplicado en el presente trabajo, HPLC Ms-Ms provee un $100 \%$ de exactitud en la confirmación diagnóstica de la presencia del carbofuran, pero otros métodos analíticos más accesibles (cromatografía en capa fina TLC, cromatografía gaseosa GC ${ }^{12}$, HPLC- UV ${ }^{22}$ ), son también válidos.

Entre los animales de compañía, el perro es la especie más frecuentemente involucrada en episodios de intoxicaciones ${ }^{1}$. En Bélgica, constituyen aproximadamente el $20 \%$ de todos los casos confirmados de envenenamiento, seguidos de gatos y caballos (11\% y 1,1\% respectivamente). En Francia, los caninos suman el $35 \%$ de los 1.500 casos que se registran anualmente, seguidos de los gatos (12\%) y bovinos (5\%). En España, los perros representan el $52-60 \%$ de los casos de envenenamiento. La mayoría de los casos de intoxicaciones de perros en Francia involucran plaguicidas $(39 \%)^{1}$. Los envenenamientos accidentales debidos al uso legal y adecuado de plaguicidas son escasos, al igual que el uso inadecuado por concentraciones elevadas. La mayoría de las intoxicaciones se producen por la colocación de cebos envenenados con el fin de matar animales, como ocurrió en el presente trabajo.

En los animales domésticos de campo o silvestres, se producen intoxicaciones secundarias como consecuencia de su ingestión por parte de animales "carroñeros" ${ }^{17,19}$. En este contexto, las especies domésticas son centinelas potenciales del uso de venenos para matar animales silvestres, ya que se han detectado los mismos principios activos en cadáveres de especies silvestres y domésticas ${ }^{15}$. No se dispone de publicaciones que registren datos similares para nuestro país.

Es importante acentuar el control de la comercialización de plaguicidas altamente ricos en principios activos y bajas DL50 ${ }^{17}$. La DL50 de algunos plaguicidas es tan baja, que el porcentaje de tales ingredientes activos en las formulaciones comerciales puede determinar ampliamente el riesgo de envenenamientos intencionados, como es el caso del carbofuran ${ }^{15}$.

A manera de conclusión, se enfatiza la importancia de promover como herramienta de diagnóstico en la práctica veterinaria, la medición de $\mathrm{BChE}$ en animales intoxicados, así como en salud pública promover la denuncia obligatoria y registro centralizado de la intoxicación por plaguicidas en animales, para aportar a la fiscalización de la venta de estos productos y a la educación a conciencia del riesgo de uso de los mismos en consideración de la salvaguarda de la salud animal y humana como bien primario. En la práctica veterinaria resulta necesario llevar a cabo un correcto relevamiento toxicológico para esclarecer la frecuencia de intoxicaciones animales.

Agradecimientos. Al CONICET, Universidad Juan Agustín Maza y DICyT Mendoza por el finaciamiento del trabajo. A la Dra. Raquel Fernandez por la supervisión de la pericia toxicológica. A los propietarios de los perros que fueron investigados y a sus médicos veterinarios por la colaboración prestada.

\section{REFERENCIAS}

1. Berny P, Colony F, Croubels S, Sachana M, Vandenbroucke V, Davanz F, Guitart R. 2010. Animal poisoning in Europe. Part 2: Companion animals. Vet $J$ 183: 255259.

2. Cabrera A, Varela W. 2009. Intoxicación por organofosforados (revisión del diagnóstico y manejo). Rev. Méd Cos Cen 588: 161-167.

3. Čolović MB, Krstić DZ, Lazarević TD, Bondžić AM, Vasić VM. 2013. Acetylcholinesterase inhibitors: Pharmacology and toxicology, Curr Neuropharmacol 11: 315-335.

4. Evans GO. 1990. Animal clinical chemistry: a practical handbook for toxicologists and biomedical researchers, 2nd ed., CRC Press, Boca Raton, Florida, p. 248.

5. Groner E, Ashani Y, Schorer D, Sterling J, Herzig Y, Weinstock M. 2007. The kinetics of inhibition of human acetylcholinesterase and butyrylcholinesterase by two series of novel carbamates. Mol Pharmacol 71: 1610-1617.

6. Guitart R, Mañosa S, Guerrero X, Mateo R. 1999. Animal poisonings: the 10 -year experience of a veterinary analytical toxicology laboratory. Vet Human Toxicol 41: 331-335.

7. Guitart R, Sachana M, Caloni F, Croubels S, Vandenbroucke V, Berny P. 2010. Animal poisoning in Europe. Part 3: Wildlife. Vet J 183: 260-265.

8. Gupta RC. 2012. Carbamates Insecticides. The Merck veterinary manual, http://www.merckmanuals.com

9. Gupta RC. 2012. Organophosphates. The Merck veterinary manual, http://www.merckmanuals.com

10. Hazardous Substances Data Bank (HSDB). 2014. Data base of National Library of Medicine's Toxnet System. http://toxnet.nlm.nih.gov

11. Hopper K, Aldrich J, Haskins SC. 2002. The recognition and treatment of the intermediate syndrome of organophosphate poisoning in a dog. $J$ Vet Emerg Crit Care 12: 99-103.

12. Karunarathna WD, Udugampala US. 2013. Carbofuran concentrations in blood, bile and tissues in fatal cases of homicide and suicide. Forensic Sci Int 227: 106-110. 
13. Luo C, Tong M, Chilukuri N, Brecht K, Maxwell DM. 2007. An in vitro comparative study on the reactivation of nerve agent-inhibited guinea pig and human acetylcholinesterases by oximes. US Army Research 42, http://digitalcommons.unl.edu/usarmyresearch/42

14. Machín DG. 1998. Curso de auto-instrucción en diagnóstico, tratamiento y prevención de intoxicaciones agudas causadas por plaguicidas. Unidad 2: Plaguicidas de tipo organofosforados y carbamatos. http://bvs.per.paho.org/ tutorial2/e/unidad2/index.html

15. Martínez-Haro M, Mateo R, Guitart R, Soler F, Pérez M, Mojicad P, García EJ. 2008. Relationship of the toxicity of pesticide formulations and their commercial restrictions with the frequency of animal poisonings. Ecotox Environ Safety 69: 396-402.

16. Pesticide Action Network (PAN Internacional). 2014. Lista de plaguicidas altamente peligrosos. http://www.rapal.org/libros_files/Lista_HHP_PAN.jpg

17. Sánchez Barbudo IS, Camarero P, Mateo R. 2012. Intoxicaciones intencionadas y accidentales de fauna silvestre y doméstica en España: diferencias entre comunidades autónomas. Rev Toxicol 29: 20-28.
18. SENASA (Servicio Nacional de Sanidad y Calidad Agroalimentaria). http://www.senasa.gov.ar

19. Soler Rodriguez F, Oropesa AL, Pérez M. 2006. Análisis de los envenenamientos en fauna silvestre. Situación en Extremadura. Rev Toxicol 23: 35-38.

20. Talcott P, Peterson M. 2006. Small Animal Toxicology, Elsevier, St. Louis, 930 p.

21. Tiwari RM, Sinha M. 2010. Veterinary Toxicology, Oxford Book Company, Jaipur (India), p.17-38.

22. Vinod K, Naidu NV. 2013. Development and validation of RP-HPLC method for the analysis of carbofuran and in its formulations Der Pharma Chemica 5: 313-319.

23. World Health Organization (WHO). 2009. The WHO recommended classification of pesticides by hazard and guidelines to classification. Ed. Wissenchaftliche Verlagsgesellschaftmb mbH. Stuttgart. Alemania, http://www. who.int/entity/ipcs /publications/pesticides2009_cover.jpg

\section{Revista Veterinaria ingresa a SciELO}

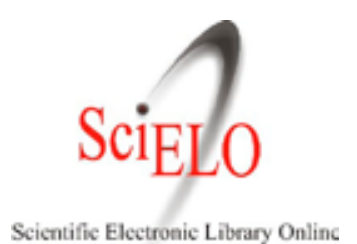

Scientific Electronic Library Onlinc

Revista Veterinaria, publicación oficial de la Facultad de Ciencias Veterinarias de la Universidad Nacional del Nordeste (Corrientes, Argentina), ha logrado acceder al Núcleo Básico de Revistas Científicas Argentinas (Nivel 1), luego de calificar adecuadamente en el Centro Argentino de Información Científica y Tecnológica (CAICYT), según Resolución 2485/14 del Consejo Nacional de Investigaciones Científicas y Técnicas (CONICET).

Sobre un puntaje máximo de 33 se obtuvieron 32 puntos. Tal calificación constituye "una garantía de la excelencia de la publicación" (sic) y queda expedita la vía del Portal SciELO (Scientific Electronic Library Online) para los artículos publicados.

En tal calificación gravitó positivamente la circunstancia de haber aumentado el índice de impacto (Scimago-Elsevier) y haber disminuido las autocitaciones. También se tuvieron en cuenta aspectos como la amplia cobertura de la revista, la calidad científica del Comité Editorial, los criterios de evaluación de los artículos, el origen de los autores (locales $60 \%$, nacionales $13 \%$, extranjeros $26 \%$, en idioma inglés), el adecuado balance entre trabajos científicos originales y reseñas bibliográficas (ambos con alta calidad), así como el estricto cumplimiento de la periodicidad semestral y la favorable acogida por indizadores como Cab, Doaj, Ebsco, Gale Cengage, Infocyt, Latindex y Scopus.

Se consolida de esta manera la continuidad de "Revista Veterinaria", que en el año próximo (2015) cumplirá 50 años de existencia, en coincidencia con el $95^{\circ}$ aniversario de la creación de la Facultad. 